\title{
Caracterización del neonato asistido con ventilación mecánica. Hospital “Abel Santamaría” Agosto 2009 - Marzo 2013
}

\author{
Adrián Naranjo', Gabino Arman², Agustín Montano ${ }^{3}$, Yorelvis Haces Trujillo4
}

\section{RESUMEN}

Objetivo: Caracterizar al neonato con ventilación mecánica en el Hospital “Abel Santamaría”, en Pinar del Río, Cuba. Material y Métodos: Estudio observacional, transversal y descriptivo; realizado entre agosto de 2009 y marzo de 2013. Un total de 164 neonatos con ventilación mecánica fueron estudiados en la Unidad Neonatal Intensiva del Hospital Abel Santamaría. Se analizó: edad gestacional, peso al nacer, historia médica de la madre, causas de la ventilación y complicaciones de la ventilación.

Resultados: El 62 \% fue de sexo masculino, la edad gestacional que predominó fue de más de 32 semanas, el 37 \% de los neonatos tuvieron menos de $2500 \mathrm{~g}$. La Hipertensión inducida por la gestación estuvo presente como antecedente materno en el $17 \%$ de los bebés ventilados, la causa más común para ventilación fue el Síndrome de distréss respiratorio grave (SDRG), la disfunción digestiva se presentó en el 14 \% como complicación, la hemorragia pulmonar fue la mayor causa de muerte.

Conclusiones: A menor tiempo de ventilación mecánica, mayor supervivencia. La hemorragia pulmonar fue la principal causa de muerte. (Horiz Med 2013; 13(1): 24-30)

Palabras clave: ventilación mecánica, neonato, cuidado neonatal. (Fuente: DeCS BIREME).

Characterization of neonatal assisted mechanical ventilation. “Abel Santamaría” Hospital in August 2009 - March 2013

\section{ABSTRACT}

Objective: To characterize the mechanical ventilation in neonates of "Abel Santamaría " Hospital, in Pinar del Rio, Cuba . Material and Methods: An observational, cross-sectional, descriptive study, conducted between August 2009 and March 2013. A total of 164 mechanically ventilated infants were studied in the Neonatal Intensive Care Unit of the Hospital Abel Santamaría. We analyzed: gestational age, birth weight, medical history of the mother, causes of ventilation and complications of ventilation.

Results: $62 \%$ were male, gestational age was predominantly more than 32 weeks, 37\% of infants had less than $2500 \mathrm{~g}$. Pregnancy induced hypertension was present as maternal history in $17 \%$ of ventilated infants, the most common cause for ventilation was acute respiratory distress syndrome (ARDS), digestive dysfunction occurred in $14 \%$ as a complication, pulmonary hemorrhage was the leading cause of death.

Conclusions: The lower the duration of mechanical ventilation, the longer survival. Pulmonary haemorrhage was the leading cause of death. (Horiz Med 2013; 13(1): 24-30)

Key words: mechanical ventilation, neonatal, neonatal care. (Source: MeSH NLM).

1 Instructor no graduado de Cardiología, Estudiante de 5to año de la Carrera de Medicina.

2 Especialista de 1 er grado en Ginecobstetricia. Director de Programa de Atención Materno Infantil, Pinar del Río Cuba.

3 Instructor no graduado de Neonatología. Estudiante de 5to año de Medicina.

4 Instructor no graduado de Neonatología. Estudiante de 5to año de Medicina.

Hospital General Docente Abel Santamaría Cuadrado. Universidad de Ciencias Médicas Pinar del Río. Cuba 


\section{INTRODUCCIÓN}

La dificultad respiratoria es uno de los padecimientos más frecuentes en los recién nacidos pretérmino y está íntimamente relacionada con problemas del desarrollo pulmonar, trastornos de la adaptación respiratoria tras el nacimiento, patologías infecciosas, trastornos de otros sistemas como: anemia, hipotermia, asfixia perinatal, afecciones cardiovasculares, y es sin duda, la que más aporta a las Unidades de Cuidados Intensivos Neonatales (UCIN) y a la ventilación mecánica en el neonato. $(1,2)$

En la actualidad, la ventilación asistida en el neonato, constituye una terapia indispensable en las unidades de cuidados intensivos neonatales.

La ventilación temprana suele ser útil, sobre todo en el recién nacido pretérmino con demanda de oxígeno elevada y una capacidad de reserva pulmonar y energética disminuida; el inicio temprano de la ventilación mecánica contribuye a evitar la insuficiencia respiratoria (1). Muchas de las enfermedades del neonato tratadas en la terapia intensiva requieren de ventilación temprana. La indicación para la ventilación mecánica en el recién nacido está condicionada por la patología de base y no debe esperarse que presente manifestaciones o signos de insuficiencia respiratoria, ya sea gasométrica o clínica. Si conocemos bien las diferentes patologías, su fisiopatología, la posibilidad de complicación, la posibilidad de secuelas para el neonato, la indicación será oportuna y rápida. El $30 \%$ de los niños ventilados en los Estados Unidos es por la membrana hialina $(\mathrm{MH}) \mathrm{y}$, entre el 50 y el $70 \%$ de éstos, son pretérmino (3).

Con el uso de surfactante exógeno y la maduración pulmonar intrauterina, la incidencia de la $\mathrm{MH}$ ha disminuido, no obstante se ventila entre el 40 al 60 $\%$ de los niños nacidos asfícticos (4).

La mortalidad neonatal ha descendido progresivamente en los últimos años, gracias al desarrollo de novedosas técnicas relacionadas con la ventilación mecánica. Uno de cada tres nacidos vivos que ingresan en las unidades de cuidados intensivos neonatales (UCIN) requiere ventilación mecánica por diversas afecciones (5). Su uso temprano suele ser útil pues sobre todo con ello se evita una insuficiencia respiratoria grave. Por lo que es de vital importancia conocer, las características de la ventilación mecánica, en neonatos atendidos en la Unidad de Cuidados Intensivos Neonatales del Hospital Abel Santamaría.

\section{MATERIAL Y MÉTODOS}

Estudio observacional, descriptivo y transversal, en neonatos asistidos con ventilación mecánica en la Unidad de Cuidados Intensivos Neonatales del Hospital Abel Santamaría entre agosto 2009 y marzo 2013.

El universo estuvo constituido por todos los pacientes en los que fue necesario el uso de ventilación mecánica, constituyendo este grupo 164 neonatos, Se estudió todo el universo, no requiriendo criterios muestrales.

Se estudió las siguientes variables: Sexo, peso, edad gestacional, antecedentes maternos, causa de ventilación, complicaciones durante la ventilación, tiempo total de ventilación y estado vital al retirar la ventilación

El estudio fue aprobado por el Comité de Ética del Hospital General Docente Abel Santamaría Cuadrado.

\section{RESULTADOS}

En cuanto a sexo y edad gestacional, por grupos de peso (Tabla 1), se encontró un predominio del sexo masculino (62\%). La edad gestacional de más de 32 semanas (37\%) fue significativamente la más frecuente, con un predominio del grupo de peso menor de $2500 \mathrm{~g}$.

Al relacionar los antecedentes maternos con las causas de ventilación mecánica (Tabla 2), en el $30 \%$ de los neonatos ventilados no se halló antecedentes maternos importantes. La toxemia estuvo presente como antecedente materno en el $17 \%$ de los bebés ventilados, junto con el meconio, partos múltiples y la sepsis ovular. 
Tabla 1. Sexo de los recién nacidos ventilados y edad gestacional según grupos de peso.

\begin{tabular}{|c|c|c|c|c|c|c|c|c|}
\hline \multicolumn{9}{|c|}{ GRUPOS DE PESO } \\
\hline \multirow[t]{2}{*}{ VARIABLES } & \multicolumn{2}{|c|}{$<2500 \mathrm{~g}$} & \multicolumn{2}{|c|}{$2500-3499 \mathrm{~g}$} & \multicolumn{2}{|c|}{$>3500 \mathrm{~g}$} & \multicolumn{2}{|c|}{ Total } \\
\hline & No & $\%$ & No & $\%$ & No & $\%$ & No & $\%$ \\
\hline \multicolumn{9}{|l|}{ SEXO } \\
\hline MASCULINO & 60 & 37 & 34 & 21 & 7 & 4 & 101 & 62 \\
\hline FEMENINO & 46 & 28 & 16 & 9 & 1 & 1 & 63 & 38 \\
\hline \multicolumn{9}{|c|}{ EDAD GESTACIONAL (SEMANAS) } \\
\hline $26-29,6$ & 20 & 12 & 0 & - & 0 & - & 20 & 12 \\
\hline $30-31,6$ & 25 & 15 & 0 & - & 0 & - & 25 & 15 \\
\hline 32 y más & 61 & 37 & 50 & 31 & 8 & 5 & 119 & 73 \\
\hline
\end{tabular}

Tabla 2. Relación entre antecedentes maternos y causa de ventilación

\begin{tabular}{|c|c|c|c|c|c|c|c|c|c|c|c|c|c|c|}
\hline \multirow{3}{*}{$\begin{array}{l}\text { ANTECEDENTES } \\
\text { MATERNOS }\end{array}$} & \multicolumn{12}{|c|}{ CAUSA DE VENTILACIÓN } & \multirow{2}{*}{\multicolumn{2}{|c|}{ TOTAL }} \\
\hline & \multicolumn{2}{|c|}{ ASFIXIA } & \multicolumn{2}{|c|}{ BNM $^{*}$} & \multicolumn{2}{|c|}{ APNEA } & \multicolumn{2}{|c|}{ SDRG } & \multicolumn{2}{|c|}{ SDRT } & \multicolumn{2}{|c|}{$\mathrm{CC}$} & & \\
\hline & No. & $\%$ & No. & $\%$ & No. & $\%$ & No. & $\%$ & No. & $\%$ & No. & $\%$ & No. & $\%$ \\
\hline PARTO MÚLTIPLE & 0 & - & 3 & 17 & 2 & 24 & 11 & 19 & 7 & 18 & 0 & - & 23 & 14 \\
\hline MECONIO & 15 & 47 & 0 & - & 0 & - & 4 & 7 & 5 & 12 & 0 & - & 24 & 15 \\
\hline SEPSIS OVULAR & 3 & 10 & 5 & 28 & 1 & 13 & 9 & 15 & 4 & 10 & 0 & _ & 22 & 13 \\
\hline $\begin{array}{l}\text { DIABETES } \\
\text { MELLITUS }\end{array}$ & 0 & - & 1 & 6 & 1 & 13 & 4 & 7 & 1 & 2 & 1 & 17 & 8 & 5 \\
\hline $\begin{array}{l}\text { HIPERTENSIÓN } \\
\text { ARTERIAL }\end{array}$ & 0 & - & 0 & - & 2 & 24 & 5 & 8 & 2 & 5 & 1 & 17 & 10 & 6 \\
\hline TOXEMIA & 4 & 13 & 3 & 17 & 1 & 13 & 10 & 17 & 10 & 24 & 0 & - & 28 & 17 \\
\hline NINGUNA & 10 & 30 & 6 & 32 & 1 & 13 & 16 & 27 & 12 & 29 & 4 & 66 & 49 & 30 \\
\hline TOTAL & 32 & 100 & 18 & 100 & 8 & 100 & 59 & 100 & 41 & 100 & 6 & 100 & 164 & 100 \\
\hline
\end{tabular}

En la tabla 3, se observan las causas de ventilación mecánica por grupos de peso. La causa más común fue el Síndrome de distress respiratorio grave (SDRG), con $36 \%$ de los casos, seguida del SDRT en el $25 \%$ de todos los neonatos ventilados, predominando los neonatos con menos de $2500 \mathrm{~g}$ de peso al nacer. Las cardiopatías congénitas (CC) fueron la causa que con menos frecuencia condujo a la utilización de ventilación mecánica. 
Tabla 3. Relación entre causas que motivaron la ventilación mecánica y grupos de peso.

\begin{tabular}{|c|c|c|c|c|c|c|c|c|}
\hline \multicolumn{9}{|c|}{ GRUPOS DE PESO } \\
\hline \multirow{2}{*}{$\begin{array}{l}\text { CAUSAS DE } \\
\text { VENTILACIÓN }\end{array}$} & \multicolumn{2}{|c|}{$<2500 \mathrm{~g}$} & \multicolumn{2}{|c|}{$2500-3499 \mathrm{~g}$} & \multicolumn{2}{|c|}{$>3500 \mathrm{~g}$} & \multicolumn{2}{|c|}{ Total } \\
\hline & No & $\%$ & No & $\%$ & No & $\%$ & No & $\%$ \\
\hline ASFIXIA & 4 & 4 & 23 & 46 & 5 & 61 & 32 & 20 \\
\hline APNEA & 1 & 1 & 6 & 12 & 1 & 13 & 8 & 5 \\
\hline BNM $^{1}$ & 17 & 16 & 1 & 2 & 0 & - & 18 & 10 \\
\hline SDRG $^{2}$ & 54 & 51 & 5 & 10 & 0 & - & 59 & 36 \\
\hline SDRT $^{3}$ & 29 & 27 & 11 & 22 & 1 & 13 & 41 & 25 \\
\hline$C C^{4}$ & 1 & 1 & 4 & 8 & 1 & 13 & 6 & 4 \\
\hline TOTAL & 106 & 100 & 50 & 100 & 8 & 100 & 164 & 100 \\
\hline
\end{tabular}

1 Bronconeumonía

3 Síndrome de distress respiratorio transitorio

2 Síndrome de distress respiratorio grave

4 Cardiopatía Congénita

En la tabla 4, se muestra el total de complicaciones por tiempo de ventilación. El $42 \%$ de los neonatos ventilados tuvó algún tipo de complicación. Siendo la disfunción digestiva la más frecuente (14 \%).

Tabla 4. Complicaciones surgidas durante el tiempo total de ventilación.

\begin{tabular}{|c|c|c|c|c|c|c|c|c|}
\hline \multicolumn{9}{|c|}{ GRUPOS DE PESO } \\
\hline \multirow{2}{*}{$\begin{array}{c}\text { COMPLICACIONES } \\
\text { DURANTE LA } \\
\text { VENTILACIÓN } \\
\text { MECÁNICA }\end{array}$} & \multicolumn{2}{|c|}{ Hasta $24 \mathrm{~h}$} & \multicolumn{2}{|c|}{$+24 \mathrm{~h}$ hasta $240 \mathrm{~h}$} & \multicolumn{2}{|c|}{$+240 \mathrm{~h}$} & \multicolumn{2}{|c|}{ Total } \\
\hline & No & $\%$ & No & $\%$ & No & $\%$ & No & $\%$ \\
\hline DISFUNCIÓN DIGESTIVA & 8 & 80 & 10 & 34 & 5 & 17 & 23 & 14 \\
\hline BNM & 2 & 20 & 7 & 24 & 10 & 33 & 19 & 12 \\
\hline HEMORRAGIA PULMONAR & 0 & _- & 4 & 14 & 12 & 40 & 16 & 10 \\
\hline ATELECTASIA & 0 & _- & 8 & 28 & 3 & 10 & 11 & 7 \\
\hline TOTAL & 10 & 27 & 29 & 100 & 30 & 100 & 69 & 42 \\
\hline SIN COMPLICACIONES & 43 & 81 & 45 & 60 & 7 & 18 & 95 & 58 \\
\hline TOTAL & 53 & 32 & 74 & 45 & 37 & 23 & 1 & 100 \\
\hline
\end{tabular}


En la tabla 5, se observa la mortalidad asociada a las complicaciones surgidas durante la ventilación. La hemorragia pulmonar fue la complicación durante la ventilación que más mortalidad tuvo asociada para un $62 \%$ de los casos, seguida de la BNM con un total de 8 fallecidos.

Tabla 5. Comportamiento de la mortalidad asociada a las complicaciones surgidas durante la ventilación mecánica.

\begin{tabular}{|c|c|c|c|c|c|c|c|c|c|c|c|c|}
\hline \multirow[t]{3}{*}{ MORTALIDAD } & \multicolumn{12}{|c|}{ GRUPOS DE PESO } \\
\hline & \multicolumn{2}{|c|}{$\begin{array}{c}\text { Disfunción } \\
\text { digestiva }\end{array}$} & \multicolumn{2}{|c|}{ BNM } & \multicolumn{2}{|c|}{$\begin{array}{l}\text { Hemorragia } \\
\text { pulmonar }\end{array}$} & \multicolumn{2}{|c|}{ Atelectasia } & \multicolumn{2}{|c|}{ Ninguna } & \multicolumn{2}{|c|}{ Total } \\
\hline & No & $\%$ & No & $\%$ & No & $\%$ & No & $\%$ & No & $\%$ & No & $\%$ \\
\hline VIVOS & 21 & 91 & 11 & 58 & 6 & 38 & 8 & 73 & 89 & 94 & 135 & 82 \\
\hline FALLECIDOS & 2 & 9 & 8 & 42 & 10 & 62 & 3 & 27 & 6 & 6 & 29 & 18 \\
\hline TOTAL & 23 & 100 & 19 & 100 & 16 & 100 & 11 & 100 & 95 & 100 & 164 & 100 \\
\hline
\end{tabular}

En la tabla 6, se presenta el estado al retirar la ventilación atendiendo al tiempo total que se mantuvo la misma. El $17 \%$ de todos los ventilados fallecieron, siendo más frecuente la mortalidad en los que se ventilaron por más de $240 \mathrm{~h}$ (38 \%), no siendo así en los que se mantuvieron acoplados menos de $24 \mathrm{~h}$ representando estos solo el $2 \%$ del total.

Tabla 6. Estado al retirar la ventilación según el tiempo total de ventilación.

\begin{tabular}{|c|c|c|c|c|c|c|c|c|}
\hline \multirow{3}{*}{$\begin{array}{l}\text { ESTADO AL RETIRAR } \\
\text { LA VENTILACION }\end{array}$} & \multicolumn{6}{|c|}{ GRUPOS DE PESO } & & \\
\hline & \multicolumn{2}{|c|}{ Hasta $24 \mathrm{~h}$} & \multicolumn{2}{|c|}{$+24 \mathrm{~h}$ hasta $240 \mathrm{~h}$} & \multicolumn{2}{|c|}{$+240 \mathrm{~h}$} & \multicolumn{2}{|c|}{ Total } \\
\hline & No & $\%$ & No & $\%$ & No & $\%$ & No & $\%$ \\
\hline VIVOS & 6 & 38 & 8 & 73 & 89 & 94 & 135 & 82 \\
\hline FALLECIDOS & 10 & 62 & 3 & 27 & 6 & 6 & 29 & 18 \\
\hline
\end{tabular}

\section{DISCUSIÓN}

El predominio del sexo masculino encontrado, coincide con los resultados de reportes de otros autores que señalan que muchas de las afecciones frecuentes en este tipo de niños, como la dificultad respiratoria grave del pretérmino, la enfermedad pulmonar crónica, entre otras, tienen lugar en los varones $(5,6)$. Sin embargo, algunos estudios reportan un predominio de ingresos de neonatos del sexo femenino $(5,7)$. González Cabello y Cols (8) hallaron una edad gestacional promedio de 30 semanas y un peso promedio de $1100 \mathrm{~g}$. Similar a lo reportado por Santamaría (6) en México y Acevedo (4) en Santiago de Cuba. Estudios, que no coinciden con el nuestro.

La HIG fue el antecedente predominante, seguido del meconio y del antecedente de partos múltiples, hecho que no coincide con los demás estudios consultados $(11,12)$. En más de la mitad de los neonatos asistidos con ventilación mecánica, existían antecedentes patológicos de base por parte de las madres. Coincidiendo con la mayoría de estudios realizados en América Latina y el Caribe $(13,14)$. 
Los resultados encontrados al relacionar las causas de ventilación mecánica por grupos de peso concuerdan con los reportados por otros autores. Se señalan como principales causas de asistencia respiratoria mecánica: la enfermedad de membrana hialina, la asfixia perinatal y la bronconeumonía congénita, las cuales varían en orden de frecuencia de un centro a otro (15).

Otros investigadores señalan además: apnea recurrente y falla de la presión positiva continua. $(16,17)$. Estos resultados no coinciden con nuestro estudio ya que se presentaron con mayor frecuencia el SDRG, seguido del SDRT, predominando estos en neonatos de menos de $2500 \mathrm{~g}$ de peso al nacer dato éste, que sí coincide con estudios realizados en Chile y Perú $(10,18)$.

Las complicaciones del uso de la ventilación mecánica dependen de cada centro, de las características de sus pacientes, de la experiencia del equipo médico y de los medios con que se disponga, pero también son más frecuentes en la medida en que la ventilación mecánica se prolongue en el tiempo. No coincide con esta serie, pues para muchos autores la complicación fundamental de la ventilación en estos niños fue la sepsis $(11,17)$, condicionados por la prolongada hospitalización, las complicaciones iatrogénicas relacionadas con la terapia intensiva y los estados prolongados, dada la enfermedad de base. También se reportan como complicaciones frecuentes las bronconeumonías, procesos hemorrágicos, atelectasias, persistencia del conducto arterioso y enfermedad pulmonar crónica.(19).

Es de destacar que casi todas las complicaciones aparecieron en los casos donde la ventilación mecánica se prolongó por más de $24 \mathrm{~h}$ y hasta $240 \mathrm{~h}$, no así en correspondencia con los trabaj os revisados donde las complicaciones aparecieron posterior a las 96h. En un estudio realizado en el Hospital General Docente «Iván Portuondo», San Antonio de los Baños (La Habana) las complicaciones más frecuentes asociadas a la ventilación mecánica fueron los procesos infecciosos, y la supervivencia se vio afectada por esta causa en nuestros casos de estudio (20). Aunque se presentaron pocos procesos hemorrágicos y escapes de aire, la supervivencia fue menor al compararla con otras complicaciones. Se plantean los procesos infecciosos como una de las principales complicaciones en el neonato ventilado y que la sepsis está estrechamente vinculada a muchos factores de manej o técnico y ambientales. $(20,21)$.

Para Boccarato (17), aunque encontró poca incidencia de procesos hemorrágicos y escapes aéreos, halló menor supervivencia al compararla con otras complicaciones. Ávila (18), encontró una mortalidad global por volutrauma del 6,8 \%. Y Chinea (3) encontró menor supervivencia por sepsis respiratoria y hemorragia intraventricular.

En la literatura internacional, existen pocos trabajos que hablen acerca del estado de los neonatos al retirar la ventilación. Santamaría y col. (6) encontraron en su estudio que el $49 \%$ de los neonatos ventilados por más de $120 \mathrm{~h}$ al retirarle la misma, fallecieron. En nuestro estudio, encontramos que a menor duración de la ventilación, mayor fue la supervivencia, factor señalado como muy importante por diversos autores (21).

En conclusión, se observó que en los ventilados por más de 240 horas surgieron el mayor número de complicaciones, no siendo así en los que se ventilaron por menos de $24 \mathrm{~h}$.

\section{Fuentes de financiamiento}

El estudio ha sido autofinanciado por el autor.

\section{Conflicto de interés}

El autor declara no tener ningún conflicto de interés.

\section{Correspondencia:}

Adrián Naranjo

Dirección: Calle 57 entre 70 y 72 No. $70^{\mathrm{a}} 02$ Consolación del Sur, Pinar del Río, Cuba

Teléfono: 53101674

Correo electrónico: adrian90@princesa.pri.sld.cu 


\section{REFERENCIAS BIBLIOGRÁFICAS}

1. Sola A. Recién nacido de extremadamente baja edad gestacional. Cuidados del feto y del recién nacido. Bss Ass: Ed Cient Interam 2001:1615-24.

2. Correa J, Pineros JG, Gómez A, Báez M, Andrade V, et al. Síndrome de dificultad respiratoria del recién nacido con enfermedad de membrana hialina. Actual Pediatr 2002;8(3):106-19.

3. Chinea Pentón Y, Prieto Valdés MA. Algunos factores asociados a la ventilación mecánica en el recién nacido. [Sitio en Internet]. Disponible en: http:// www. 16 deabril.sld.cu/eventos / xviiforum / presenciales/Pediatria Acceso el 28 septiembre 2006.

4. Acevedo Ortiz A, Matos Toledo AC. Asistencia Respiratoria mecánica en el recién nacido muy bajo de peso al nacer. [Sitio en Internet] Disponible en: $\quad$ http:// www. ilustrados.com/ publicaciones/ Salud Acceso el 28 septiembre 2006.

5. Bhat R. Neonatology. Edit. Saunders Co; 2005. [Sitio en Internet] Disponible en: http://www. springerlink.com/index/RJ187553X5742457.pdf Acceso el 28 septiembre 2006.

6. Santamaría R, Valencia R, Ramírez P. Supervivencia en RNMBP sometidos a ventilación mecánica. Salud Tab 2002;8(1):422-26.

7. Álvarez Urbina J. Morbimortalidad en recién nacidos muy bajo peso en el hospital San Juan de Dios, San José, Costa Rica. Acta Pediátr Costarric 2003;15(2):46-52.

8. González Cabello HJ, Flores Esqueda NB, García HJ. Sobrevida al egreso de UCIN en recién nacidos menores de 1500 gramos. [Sitio en Internet] Disponible en: http: / / www.edumed.imss.gob.mx/pediatria/Jornadas/ memorias05/resumenes/resumen70.htm Acceso el 8 mayo 2007.

9. Grupo Colaborativo NEOCOSUR. Very low birth weight outcomes in 11 South American. NCIUs J of Perinatology 2002; 22:2-7.

10. Goldenberg RL, Wright LL. Repeated courses of antenatal corticosteroids Obstet Gynecol 2003;97:316-7.

11. Lohmann GB P, Rodríguez M, et al. Mortalidad en recién nacidos de extremo bajo peso al nacer en la unidad de neonatología del Hospital Nacional Cayetano Heredia entre enero 2000 y diciembre 2004. Rev Med Hered 2006;17(3):141-7
12. Suárez Martache G. Evolución del desarrollo psicomotor en infantes que requirieron A.R.M. en el período neonatal. Acta Pediatr Costa Rica 2002;3(16):356-63.

13. Morgues $M$, Henríquez MT, Tohá $D$, Vernal $P$, et al. Sobrevida del niño menor de 1500 gramos en Chile. Rev Chil Obstet Ginecol 2002;67(2):100-5.

14. García Fernández Y, Fernández RM, Rodríguez M, Pérez E. Supervivencia en el recién nacido ventilado. Hospital General Docente «lván Portuondo», San Antonio de los Baños. Rev Cubana Pediatr. [serie en Internet] 2006; 78(4). Disponible en: http://bvs.sld.cu/revistas/ped/ vol78_04_06/ped02406.htm

15. Donn SM, Nicks JJ, Becker MA. Triggar happy: flow synchronization shortens the duration of assisted ventilation in preterm infant with respiratory distress syndrome. Rev Cubana Pediatr 2003;33(2):324-8.

16. Donn S, Sinha S. Invasive and no invasive neonatal mechanical Ventilation. Respiratory Care. Pediatrics 2003;48(4):66-72.

17. Boccarato A, Taboa S, Panizza R. Valoración de la asistencia ventilatoria en una unidad de tratamiento intensivo neonatal. Arch Pediatr Urug 2004;75(1):13-25.

18. Avila R, Yunes JL, Naranjo CY, et al. Volutrauma en recién nacidos con ventilación mecánica convencional. Archivos de Investigación Pediátrica en México. [serie en Internet] 2000;8(2). Disponible en: http://www. medinet.net. $\mathrm{mx} /$ conapeme/revistas/revista 8 / conapeme_revista8_volutrauma.htm Accedido el 8 mayo 2007.

19. Raju TN, Marshall CL, Hayashi RH, Vasa R, Vidyasagar D. Asistencia respiratoria mecánica en el recién nacido. Clínicas de Perinatología 2002;105(2):247-65

20. Arregoitía M, Tudela JM, Sellek M, González R. Mortalidad por Distres Respiratorio Idiopático. Rev Cubana Pediatr 1994; 66 (3):150-156.

21. Nodal A, Aguirre A, et al. Ventilación mecánica en el recién nacido. Experiencia de dos años de trabajo. Rev Cubana Pediatr 1990; 62 (2):271-28 